\title{
An association between $\mathrm{N}$-terminal pro-brain natriuretic protein level and risk of left ventricular hypertrophy in patients without heart failure
}

\author{
LEI HUANG $^{1}$, LONGFEI HUANG ${ }^{2}$, JING YU ${ }^{2}$, XIANMING WU ${ }^{2}$ and JINYAN ZHAO ${ }^{3}$ \\ ${ }^{1}$ Department of Cardiology, Ningbo Hangzhou Bay Hospital, Ningbo, Zhejiang 315336; \\ ${ }^{2}$ Department of Cardiology, Tianjin Medical University General Hospital, Tianjin 300052; \\ ${ }^{3}$ Department of Cardiology, Tianjin Union Medical Center, Tianjin 300121 P.R. China
}

Received August 5, 2019; Accepted February 5, 2020

DOI: $10.3892 /$ etm.2020.8598

\begin{abstract}
The objective of the present study was to investigate the association between $\mathrm{N}$-terminal-pro-brain natriuretic peptide (NT-proBNP) quartiles and the risk of left ventricular hypertrophy (LVH), as well as to assess the association between NT-proBNP and hallmarks of LVH in heart failure (HF)-negative patients. Logistic regression analysis was used to analyze four groups of participants, who were stratified according to NT-proBNP quartiles, in order to investigate the association between NT-proBNP and the risk of LVH. Subsequently, analyses involving uni- and multivariate linear regression were performed to evaluate the associations of NT-proBNP with LV mass (LVM), LVM index (LVMI) and relative wall thickness (RWT). The results indicated that the occurrence of LVH was progressively enhanced along with increasing NT-proBNP quartiles in patients without HF. The univariate logistic regression analysis revealed that the groups of quartiles 4 and 3 carried a 5.254 and 1.757 times greater risk of LVH than the group of the lowest NT-proBNP quartile, respectively. Furthermore, the multivariate logistic regression analysis indicated that, compared with the quartile 1 group, participants in quartiles 2-4 had a significantly increased risk of LVH. In addition, significant positive linear associations of Lg(NT-proBNP) with LVM and LVMI were determined, while a inverse association between $\operatorname{Lg}(\mathrm{NT}$-proBNP) and RWT was indicated. The results of the present study suggested that the risk of LVH increased progressively with increasing NT-proBNP quartiles. On the basis of these results, NT-proBNP may be an
\end{abstract}

Correspondence to: Dr Lei Huang, Department of Cardiology, Ningbo Hangzhou Bay Hospital, 1155 Binhai Second Road, Ningbo Hangzhou Bay, Ningbo, Zhejiang 315336, P.R. China

E-mail: huanglei6854@outlook.com

Key words: N-terminal pro-brain natriuretic peptide, left ventricular hypertrophy, left ventricular mass, left ventricular mass index, relative wall thickness effective independent prognostic marker for the risk of LVH in patients without HF.

\section{Introduction}

Plasma concentrations of cardiac-derived natriuretic peptides, specifically N-terminal pro-brain natriuretic peptide (NT-proBNP) and brain natriuretic peptide (BNP), have been firmly associated with cardiac function $(1,2)$. The increased release of those natriuretic peptides by cardiac myocytes into the bloodstream may be the result of increased ventricular wall stress, hypertrophy or volume overload. Therefore, measurement of the increased serum levels of these markers may have the potential to facilitate the accuracy of diagnosis and prognosis for patients with heart failure (HF), thereby improving the effectiveness of treatment strategies (3). Thus, these peptides are recognized as diagnostically and prognostically meaningful biomarkers for patients with $\mathrm{HF}$ (4).

Previous studies have also suggested that NT-proBNP may be an effective biomarker to diagnose left ventricular hypertrophy (LVH). To date, numerous studies have aimed to determine the associations of various natriuretic peptide levels with the risk of LVH in cardiovascular disease $(5,6)$. Most studies have focused on the association between natriuretic peptides and the diagnostic indices measured by echocardiography, particularly left ventricular diastolic dysfunction and left ventricular mass (LVM) index (LVMI) (7-9).

While the prognostic significance of the NT-proBNP regarding the risk of $\mathrm{LVH}$ has been evaluated in multiple research studies, the association of NT-proBNP quartiles with the risk of $\mathrm{LVH}$ in HF-negative patients remains to be explored. In addition, the association between the serum levels of NT-proBNP with measures of LVH, including LVMI, LVM and relative wall thickness (RWT), has been insufficiently investigated or reported. Therefore, the purpose of the present study was to determine how NT-proBNP levels, assessed in different quartiles, may affect the occurrence of $\mathrm{LVH}$ and to investigate the association between NT-proBNP and measures of LVH, including LVM, LVMI and RWT, in HF-negative patients. 


\section{Patients and methods}

Patients. As the present study was a retrospective observational study, the requirement for patient consent and specifically ethical approval was waived. Medical records of 774 patients who received standard treatment at the Department of Cardiology of Tianjin Medical University General Hospital (Tianjin, China) between June 2015 and September 2015 were reviewed. The following exclusion criteria were then applied to the sample pool: i) Lack of critical clinical data; ii) serious primary conditions, including liver and renal failure, iii) other critical illnesses. The criteria for HF-negative status were established as the absence of any symptom that may contribute to the diagnosis of acute HF (AHF) or chronic HF (CHF). In terms of AHF, the diagnosis was confirmed on the basis of chest X-ray, which indicated cardiac congestion, as well as echocardiography revealing cardiac dysfunction and abnormally upregulated cardiac markers (10). In terms of CHF, in addition to the features of cardiac dysfunction, particularly echocardiography suggesting systolic or diastolic dysfunctions, the confirmative symptoms also included certain other somatic abnormalities, including swollen ankle, respiratory arrest and fatigue in a stationary body position or along with movements (10). Taking all of the above standards into account, 622 patients (age range, $16-89$ years; mean age, $61.5 \pm 13$ years; 366 males and 296 females) were finally included in the present study.

Assays for biochemical indices and NT-proBNP. The biochemical indices and NT-proBNP were available from the patients' medical records. The relevant detection methods are described below. Directly after fasting for $10 \mathrm{~h}$, early in the morning, venipuncture of the antecubital vein was performed to collect a blood sample. All of the patient samples were delivered to the professional diagnostic laboratory for blood tests, including triglycerides (TG), total cholesterol (TC), high-density lipoprotein cholesterol (HDL-C), low-density lipoprotein cholesterol (LDL-C), uric acid (UA), alanine aminotransferase (ALT), fasting plasma glucose (FPG) and serum creatinine (Scr). Serum NT-proBNP levels were measured with an available immunoassay analyzer (Elecsys 2010; Roche Diagnostics). The quality control and quality assurance were in accordance with a standardized protocol and all the relevant parameters passed the professional assessments by the Clinical Laboratory of Tianjin Medical University General Hospital. The Clinical Laboratory of Tianjin Medical University General Hospital supplied the normal reference values.

Echocardiography. A cardiology professional consistently performed all transthoracic echocardiography measurements. Using M-mode echocardiography, the cardiac indices were measured following standardized procedures from the American Society of Echocardiography, including ventricular internal dimension in end-diastole (LVIDd), interventricular septum thickness (IVST) in end-diastole and posterior wall thickness (PWT) in end-diastole (11). Based on the measurements of the above indices, the LVM was calculated depending on the Devereux formula: $\mathrm{LVM}(\mathrm{g})=1.04 \mathrm{x}\left[(\mathrm{LVIDd}+\mathrm{IVST}+\mathrm{PWT})^{3}-\mathrm{LV}\right.$ $\left.\mathrm{IDd}^{3}\right]-13.6 \mathrm{~g}$. The calculation of LVMI was in accordance with the following formula: LVMI $\left(\mathrm{g} / \mathrm{m}^{2}\right)=\mathrm{LVM} /$ body surface area, while $L V H$ was accordingly defined as LVMI $\geq 125 \mathrm{~g} / \mathrm{m}^{2}$ in males and $\geq 120 \mathrm{~g} / \mathrm{m}^{2}$ in females (12). The calculation of the relative wall

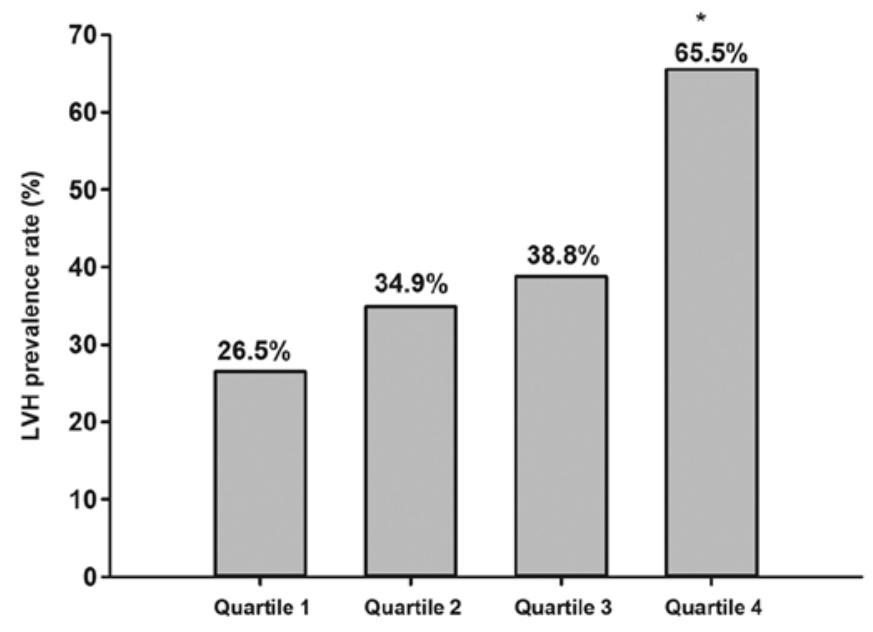

Figure 1. Association between NT-proBNP and prevalence rate of LVH. Patients were classified into four subgroups depending on serum NT-proBNP: Quartile 1 ( $\leq 56.67 \mathrm{pg} / \mathrm{ml})$, quartile $2(56.95-119.50 \mathrm{pg} / \mathrm{ml})$, quartile 3 $(119.70-414.00 \mathrm{pg} / \mathrm{ml})$ and quartile $4(\geq 415.60 \mathrm{pg} / \mathrm{ml})$. The prevalence rate of LVH increased progressively across individual NT-proBNP quartiles in patients without heart failure. ${ }^{*} \mathrm{P}<0.05$ vs. quartile 1 . NT-proBNP, N-terminal pro-brain natriuretic peptide; $\mathrm{LVH}$, left ventricular hypertrophy.

thickness (RWT) was as follows: RWT=2xPWT/LVIDd (11). The RWT partition value was set at 0.45 (13). All subjects were stratified into four groups depending on LV characteristics: Normal LV geometry (normal LVMI and RWT), concentric LV remodeling (normal LVMI and increased RWT), eccentric LVH (increased LVMI and normal RWT) or concentric LVH (increased LVMI and RWT) (14).

Statistical analysis. Statistical analyses were performed with SPSS 17.0 software (SPSS, Inc.). Continuous variables are presented as the mean \pm standard deviation or as the median with interquartile range for those variables with a skewed distribution (assessed by Kolmogorov-Smirnov test), while categorical variables are presented as numbers and percentages. Differences between groups were analyzed using an independent-samples t-test, as well as a non-parametric test (Mann-Whitney U test) for sets of measurement data or the $\chi^{2}$ test for sets of categorical data. To normalize the concentration distribution, the logarithmic values of serum NT-proBNP levels were calculated. Accordingly, the association between Log(NT-proBNP) and LVM, LVMI and RWT were analyzed using multivariate linear regression. For influencing factors, including age, sex, hypertension, percutaneous coronary intervention (PCI), diabetes, smoking, heart rate (HR), body mass index (BMI), systolic blood pressure (SBP), diastolic blood pressure (DBP), Scr, TC, TG, HDL-C, LDL-C, FPG, ALT and UA, corresponding adjustments were made. Using logistic regression analysis, the association between NT-proBNP quartiles and the risk of LVH was interpreted. Risk ratios (RRs) were presented with $95 \%$ CIs. All of the statistical comparisons were two-tailed and $\mathrm{P}<0.05$ was considered to indicate statistical significance.

\section{Results}

The occurrence of LVH increases progressively along with individual NT-proBNP quartiles in HF-negative patients without 
Table I. Clinical characteristics of the study subjects in N-terminal pro-brain natriuretic peptide quartiles.

\begin{tabular}{|c|c|c|c|c|c|c|}
\hline Item & $\begin{array}{c}\text { Total } \\
(n=622)\end{array}$ & $\begin{array}{c}\text { Quartile } 1 \\
\leq 56.67 \mathrm{pg} / \mathrm{ml} \\
\quad(\mathrm{n}=166)\end{array}$ & $\begin{array}{c}\text { Quartile 2 } \\
56.95-119.50 \\
\mathrm{pg} / \mathrm{ml}(\mathrm{n}=166)\end{array}$ & $\begin{array}{c}\text { Quartile 3 } \\
119.70-414.00 \\
\mathrm{pg} / \mathrm{ml}(\mathrm{n}=165)\end{array}$ & $\begin{array}{c}\text { Quartile } 4 \\
\geq 415.60 \mathrm{pg} / \mathrm{ml} \\
(\mathrm{n}=165)\end{array}$ & $\mathrm{P}$-value \\
\hline Male sex $(\%)$ & $366(55.3)$ & $102(61.4)$ & $81(48.8)$ & $90(54.5)$ & $93(56.4)$ & 0.139 \\
\hline Age (years) & $61.48 \pm 12.85$ & $54.48 \pm 12.64$ & $59.60 \pm 11.75$ & $63.81 \pm 11.75$ & $67.97 \pm 11.15$ & $<0.001$ \\
\hline Hypertension (\%) & $446(71.7)$ & $113(68.1)$ & $108(65.1)$ & 107 (64.8) & $118(71.5)$ & 0.531 \\
\hline PCI $(\%)$ & $193(31.0)$ & $30(18.1)$ & $36(21.7)$ & $56(33.9)$ & $71(43.0)$ & 0.613 \\
\hline Diabetes (\%) & $171(27.5)$ & $38(22.9)$ & $41(24.7)$ & $45(27.3)$ & $47(28.5)$ & 0.613 \\
\hline Smokers (\%) & $287(46.1)$ & $76(45.8)$ & $67(40.4)$ & $69(41.8)$ & $75(45.5)$ & 0.692 \\
\hline HR (bpm) (\%) & $72.28 \pm 15.19$ & $72.20 \pm 11.01$ & $69.84 \pm 12.96$ & $69.07 \pm 14.57$ & $77.98 \pm 19.46$ & $<0.001$ \\
\hline $\operatorname{BMI}\left(\mathrm{kg} / \mathrm{m}^{2}\right)$ & $25.71 \pm 3.56$ & $26.56 \pm 3,60$ & $25.34 \pm 3.53$ & $25.46 \pm 3.53$ & $25.53 \pm 3.48$ & 0.006 \\
\hline $\mathrm{SBP}(\mathrm{mmHg})$ & $137.76 \pm 47.11$ & $136.50 \pm 18.99$ & $137.88 \pm 18.69$ & $137.35 \pm 20.31$ & $137.36 \pm 23.79$ & 0.924 \\
\hline DBP (mmHg) & $80.44 \pm 13.58$ & $83.52 \pm 12.94$ & $80.21 \pm 13.46$ & $80.36 \pm 12.46$ & $77.72 \pm 14.85$ & 0.002 \\
\hline $\begin{array}{l}\text { Scr }(\text { umol/l) } \\
(62-133)^{\mathrm{a}}\end{array}$ & $72.60 \pm 35.95$ & $66.23 \pm 14.53$ & $71.17 \pm 15.07$ & $72.52 \pm 20.42$ & $80.71 \pm 37.10$ & 0.003 \\
\hline $\begin{array}{l}\mathrm{TC}(\mathrm{mmol} / \mathrm{l}) \\
(3.59-5.17)^{\mathrm{a}}\end{array}$ & $4.36 \pm 1.05$ & $4.40 \pm 0.94$ & $4.4 .26 \pm 0.95$ & $4.36 \pm 0.1 .08$ & $4.44 \pm 1.22$ & 0.495 \\
\hline $\begin{array}{l}\text { TG }(\mathrm{mmol} / \mathrm{l}) \\
(0.57-1.71)^{\mathrm{a}}\end{array}$ & $1.60 \pm 1.24$ & $1.75 \pm 1.26$ & $1.48 \pm 1.10$ & $1.56 \pm 1.04$ & $1.62 \pm 1.51$ & 0.272 \\
\hline $\begin{array}{l}\operatorname{HDL}(\mathrm{mmol} / \mathrm{l}) \\
(0.80-2.20)^{\mathrm{a}}\end{array}$ & $1.07 \pm 0.33$ & $1.04 \pm 0.26$ & $1.13 \pm 0.43$ & $1.08 \pm 0.33$ & $1.05 \pm 0.28$ & 0.050 \\
\hline $\begin{array}{l}\mathrm{LDL}(\mathrm{mmol} / \mathrm{l}) \\
(1.33-3.36)^{\mathrm{a}}\end{array}$ & $2.61 \pm 0.89$ & $2.62 \pm 0.82$ & $2.49 \pm 0.82$ & $2.60 \pm 0.89$ & $2.73 \pm 1.02$ & 0.109 \\
\hline $\begin{array}{l}\text { FPG }(\mathrm{mmol} / \mathrm{l}) \\
(3.6-5.8)^{\mathrm{a}}\end{array}$ & $5.82 \pm 2.03$ & $5.44 \pm 1.62$ & $5.56 \pm 1.62$ & $5.76 \pm 1.84$ & $6.50 \pm 2.68$ & $<0.001$ \\
\hline $\begin{array}{l}\operatorname{ALT}(\mathrm{U} / \mathrm{l}) \\
(5-40)^{\mathrm{a}}\end{array}$ & $25.95 \pm 38.72$ & $25.96 \pm 18.7$ & $23.81 \pm 20.38$ & $23.99 \pm 20.80$ & $26.85 \pm 21.83$ & 0.538 \\
\hline $\begin{array}{l}\mathrm{UA}(\mu \mathrm{mol} / \mathrm{l}) \\
(140-414)^{\mathrm{a}}\end{array}$ & $356.13 \pm 108.82$ & $362.45 \pm 105.91$ & $341.44 \pm 91.99$ & $350.25 \pm 97.81$ & $371.82 \pm 122.13$ & 0.046 \\
\hline LVIDd (mm) & $48.59 \pm 4.57$ & $47.53 \pm 3.66$ & $47.93 \pm 4.15$ & $48.33 \pm 4.42$ & $50.54 \pm 5.31$ & $<0.001$ \\
\hline IVST (mm) & $10.37 \pm 1.59$ & $10.29 \pm 1.33$ & $10.11 \pm 1.26$ & $10.38 \pm 1.53$ & $10.70 \pm 2.07$ & 0.008 \\
\hline PWT (mm) & $10.26 \pm 1.29$ & $10.20 \pm 1.25$ & $10.06 \pm 1.19$ & $10.24 \pm 1.20$ & $10.52 \pm 1.44$ & 0.011 \\
\hline LVM (g) & $215.60 \pm 59.91$ & $205.57 \pm 52.31$ & $203.84 \pm 52.13$ & $212.67 \pm 55.18$ & $240.54 \pm 71.26$ & $<0.001$ \\
\hline $\operatorname{LVMI}\left(\mathrm{g} / \mathrm{m}^{2}\right)$ & $121.11 \pm 31.02$ & $111.28 \pm 23.87$ & $114.66 \pm 25.52$ & $121.71 \pm 29.78$ & $136.87 \pm 37.10$ & $<0.001$ \\
\hline RWT & $0.42 \pm 0.06$ & $0.43 \pm 0.05$ & $0.42 \pm 0.06$ & $0.42 \pm 0.05$ & $0.42 \pm 0.06$ & 0.376 \\
\hline
\end{tabular}

Values are expressed as the mean \pm standard deviation or $\mathrm{n}(\%)$. ${ }^{\mathrm{a}}$ Normal ranges of laboratory parameters as indicated by the Clinical Laboratory of Tianjin Medical University General Hospital. PCI, percutaneous coronary intervention; HR, heart rate; BMI, body mass index; SBP, systolic blood pressure; DBP, diastolic blood pressure; Scr, serum creatinine; TC, total cholesterol; TG, triglycerides; HDL-C, high-density lipoprotein cholesterol; LDL-C, low-density lipoprotein cholesterol; FPG, fasting plasma glucose; ALT, alanine aminotransferase; UA, uric acid; LVIDd, LV internal dimension in end-diastole; IVST, interventricular septum thickness; PWT, posterior wall thickness; LVM, left ventricular mass; LVMI, LVM index; RWT, relative wall thickness.

$H F$. To confirm the prognostic significance of NT-proBNP levels regarding the prevalence of $\mathrm{LVH}$ in patients without $\mathrm{HF}$, they were stratified into groups of quartiles based on the NT-proBNP concentration in the present study: Quartile $1(\leq 56.67 \mathrm{pg} / \mathrm{ml})$, quartile $2(56.95-119.50 \mathrm{pg} / \mathrm{ml})$, quartile $3(119.70-414.00 \mathrm{pg} / \mathrm{ml})$ and quartile 4 ( $\geq 415.60 \mathrm{pg} / \mathrm{ml})$. The basic clinical features of the testing groups are listed in Table I. Among the four groups, the following parameters were comparable without any significant variation: Sex, smoking history, previously diagnosed PCI, hypertension and diabetes. Age, HR, FPG, LVIDd, LVM and LVMI were statistically significant among the four groups. The number of patients with $\mathrm{LVH}$ in quartiles 1-4 were 44, 58, 64 and 108, respectively. The LVH prevalence rates in NT-proBNP quartiles $1-4$ were $28.5,34.9,38.8$ and $65.5 \%$, respectively, as presented in Fig. 1. The patients in quartile 4 had an increased LVH occurrence rate in comparison with that in the other quartiles and the LVH risk increased across the individual quartiles with a positive association with the NT-proBNP level. 
Table II. Uni- and multivariate logistic regression models describing the risk for the prevalence rate of LVH in the study subjects.

\begin{tabular}{|c|c|c|c|c|}
\hline \multirow[b]{2}{*}{ Item } & \multicolumn{2}{|c|}{ Model 1} & \multicolumn{2}{|c|}{ Model 2} \\
\hline & RR $(95 \%$ CI $)$ & P-value & $\mathrm{RR}(95 \% \mathrm{CI})$ & P-value \\
\hline \multicolumn{5}{|l|}{ NT-pro BNP quartile } \\
\hline $1(\leq 56.67 \mathrm{pg} / \mathrm{ml})$ & 1 & - & 1 & - \\
\hline $2(56.95-119.50 \mathrm{pg} / \mathrm{ml})$ & $1.489(0.931-2.382)$ & 0.097 & $1.683(1.015-2.792)$ & 0.044 \\
\hline $3(119.70-414.00 \mathrm{pg} / \mathrm{ml})$ & $1.757(1.103-2.799)$ & 0.018 & $1.800(1.071-3.025)$ & 0.027 \\
\hline $4(\geq 415.60 \mathrm{pg} / \mathrm{ml})$ & $5.254(3.281-8.413)$ & $<0.001$ & $5.679(3.225-9.999)$ & $<0.001$ \\
\hline Age (years) & & & $1.014(0.997-1.031)$ & 0.115 \\
\hline Male sex & & & $1.104(0.694-1.756)$ & 0.677 \\
\hline Hypertension & & & $1.524(1.016-2.287)$ & 0.042 \\
\hline PCI & & & $1.183(0.791-1.769)$ & 0.414 \\
\hline Diabetes & & & $1.488(0.922-2.401)$ & 0.103 \\
\hline Smoking & & & $1.637(1.085-2.470)$ & 0.019 \\
\hline HR (bpm) & & & $0.991(0.980-1.003)$ & 0.142 \\
\hline BMI $\left(\mathrm{kg} / \mathrm{m}^{2}\right)$ & & & $0.991(0.939-1.045)$ & 0.731 \\
\hline $\mathrm{SBP}(\mathrm{mmHg})$ & & & $1.000(0.996-1.004)$ & 0.975 \\
\hline $\mathrm{DBP}(\mathrm{mmHg})$ & & & $1.025(1.009-1.041)$ & 0.002 \\
\hline $\operatorname{Scr}(\mathrm{mmol} / \mathrm{l})$ & & & $0.997(0.989-1.004)$ & 0.372 \\
\hline $\mathrm{TC}(\mathrm{mmol} / \mathrm{l})$ & & & $0.451(0.187-1.090)$ & 0.077 \\
\hline $\mathrm{TG}(\mathrm{mmol} / \mathrm{l})$ & & & $1.242(0.942-1.638)$ & 0.125 \\
\hline HDL-C (mmol/l) & & & $1.486(0.555-3.976)$ & 0.430 \\
\hline LDL-C (mmol/l) & & & $2.327(0.946-5.724)$ & 0.066 \\
\hline FPG (mmol/l) & & & $1.033(0.924-1.154)$ & 0.569 \\
\hline $\operatorname{ALT}(\mathrm{U} / \mathrm{l})$ & & & $1.000(0.995-1.004)$ & 0.928 \\
\hline $\mathrm{UA}(\mu \mathrm{mol} / \mathrm{l})$ & & & $1.001(0.999-1.003)$ & 0.600 \\
\hline
\end{tabular}

Model 1: Univariate logistic regression model describing the risk for the incidence of LVH in the study subjects. Model 2: Multivariate logistic regression model adjusting for age, gender, hypertension, PCI, diabetes, smoking, HR, BMI, SBP, DBP; Scr, TC, TG, HDL-C, LDL-C, FPG, ALT and UA. $\mathrm{HR}$, heart rate; BMI, body mass index; SBP, systolic blood pressure; DBP, diastolic blood pressure; Scr, serum creatinine; TC, total cholesterol; TG, triglycerides; HDL-C, high-density lipoprotein cholesterol; LDL-C, low-density lipoprotein cholesterol; FPG, fasting plasma glucose; ALT, alanine aminotransferase; UA, uric acid; RR, risk ratio; PCI, percutaneous coronary intervention; NT-proBNP, N-terminal pro-brain natriuretic peptide.

Association between NT-proBNP quartiles and LV geometric patterns. For an in-depth analysis of the correlation between NT-proBNP quartiles and LV geometric patterns, all participants were first stratified into four groups depending on their LVMI and RWT values, namely the normal LV geometry group, concentric remodeling group, eccentric LVH group and concentric LVH group. The prevalence rates of abnormal LV geometric patterns are presented for the concentric remodeling group, eccentric LVH group, eccentric LVH group and eccentric LVH group in Fig. 2 and were 16.9, 22.3, 24.2 and $45.5 \%$ in patients with NT-proBNP levels in quartiles $1,2,3$ and 4 , respectively. The prevalence rates of eccentric $\mathrm{LVH}$ between the lowest quartile and the highest quartile differed significantly. Compared with quartile 3 , the prevalence rates of concentric remodeling and eccentric LVH were statistically different form quartile 4.

Elevated NT-proBNP predicts $L V H$ risk. The association of the NT-proBNP level with LVH risk was assessed using univariate and multivariate logistic regression (Table II). Univariate logistic regression indicated that the LVH risk values in quartiles 2, 3 and 4 were 1.489 (0.931-2.382), 1.757
(1.103-2.799) and 5.254 (3.281-8.413) fold of that in the quartile 1 , respectively. The LVH prevalence rates also significantly varied among the three quartiles, as well as between the highest and lowest quartile (quartiles 4 and 1, respectively). After the results were adjusted for age, sex, hypertension, PCI, diabetes, smoking, HR, BMI, SBP, DBP, Scr, TC, TG, HDL-C, LDL-C, FPG, ALT and UA, multivariate logistic regression analysis was performed. Using the final multivariate model, the RRs for the LVH risk of patients in quartiles 2, 3 and 4 compared with those in quartile 1 were 1.683 (1.015-2.792), 1.800 (1.071-3.025) and 5.679 (3.225-9.999), respectively. Overall, patients in quartiles 2, 3 and 4 had a significantly higher LVH prevalence rate compared with patients in quartile 1 ; in summary the results indicated that patients with elevated NT-proBNP were significantly more likely to develop LVH.

Serum NT-proBNP is positively correlated with LVM and LVMI, while it is inversely correlated with RWT. To substantiate the association between NT-proBNP and LVM, LVMI and RWT, two types of regression analyses were performed. The linear regression results indicated a positive association between $\log (\mathrm{NT}-$ proBNP) and LVM (P<0.001; Fig. 3) and 
Table III. Results of multivariate modelling for $\operatorname{Lg}(\mathrm{NT}-$ proBNP) $(\mathrm{n}=662)$.

\begin{tabular}{|c|c|c|c|c|c|c|c|c|c|}
\hline \multirow[b]{2}{*}{ Item } & \multicolumn{3}{|c|}{ RWT } & \multicolumn{3}{|c|}{ LVM } & \multicolumn{3}{|c|}{ LVMI } \\
\hline & $\beta$ & $\mathrm{t}$ & P-value & $\beta$ & $\mathrm{t}$ & $\mathrm{P}$-value & $\beta$ & $\mathrm{t}$ & $\mathrm{P}$-value \\
\hline Lg(NT-proBNP) & -0.009 & -2.138 & 0.033 & 26.388 & 7.604 & $<0.001$ & 16.674 & 8.065 & $<0.001$ \\
\hline Male sex & 0.004 & 0.667 & 0.505 & -32.883 & -6.789 & $<0.001$ & -4.167 & -1.444 & 0.149 \\
\hline Age (years) & $<0.001$ & 1.416 & 0.157 & -0.143 & -0.832 & 0.406 & 0.029 & 0.289 & 0.773 \\
\hline Hypertension & 0.024 & 4.512 & $<0.001$ & 13.170 & 3.128 & 0.002 & 6.587 & 2.626 & 0.009 \\
\hline PCI & -0.012 & -2.177 & 0.030 & -1.150 & -0.269 & 0.788 & 0.226 & 0.089 & 0.929 \\
\hline Diabetes & -0.003 & -0.478 & 0.633 & 4.511 & 0.885 & 0.376 & 5.271 & 1.736 & 0.083 \\
\hline Smoking & $<0.001$ & 0.021 & 0.983 & 13.252 & 3.051 & 0.002 & 6.514 & 2.517 & 0.012 \\
\hline HR (bpm) & $<0.001$ & 1.055 & 0.292 & 0.039 & 0.318 & 0.750 & -0.010 & -0.131 & 0.896 \\
\hline $\mathrm{BMI}\left(\mathrm{kg} / \mathrm{m}^{2}\right)$ & $<0.001$ & 0.530 & 0.596 & 4.617 & 8.252 & $<0.001$ & 0.110 & 0.330 & 0.742 \\
\hline SBP (mmHg) & $<0.001$ & 1.344 & 0.179 & 0.016 & 0.403 & 0.687 & 0.011 & 0.469 & 0.639 \\
\hline DBP (mmHg) & 0.001 & 2.943 & 0.003 & 0.501 & 3.142 & 0.002 & 0.341 & 3.587 & $<0.001$ \\
\hline $\operatorname{Scr}(\mathrm{mmol} / \mathrm{l})$ & $<0.001$ & 2.597 & 0.010 & -0.034 & -0.632 & 0.528 & -0.017 & -0.533 & 0.594 \\
\hline $\mathrm{TC}(\mathrm{mmol} / \mathrm{l})$ & 0.008 & 0.783 & 0.434 & -5.955 & -0.714 & 0.475 & -2.729 & -0.549 & 0.583 \\
\hline TG (mmol/l) & -0.003 & -1.081 & 0.280 & 1.284 & 0.529 & 0.597 & 0.400 & 0.277 & 0.782 \\
\hline HDL-C (mmol/l) & -0.014 & -1.191 & 0.234 & 1.355 & 0.141 & 0.888 & -1.782 & -0.311 & 0.756 \\
\hline LDL-C (mmol/l) & -0.011 & -1.017 & 0.310 & 6.909 & 0.815 & 0.415 & 2.985 & 0.591 & 0.555 \\
\hline FPG (mmol/l) & 0.002 & 1.185 & 0.237 & 0.947 & 0.815 & 0.416 & 0.223 & 0.322 & 0.748 \\
\hline ALT (U/l) & $<0.001$ & -0.391 & 0.696 & -0.039 & -0.820 & 0.413 & -0.008 & -0.267 & 0.789 \\
\hline $\mathrm{UA}(\mu \mathrm{mol} / \mathrm{l})$ & $<0.001$ & -0.022 & 0.982 & 0.022 & 1.119 & 0.026 & 0.014 & 1.187 & 0.236 \\
\hline
\end{tabular}

PCI, percutaneous coronary intervention; HR, heart rate; BMI, body mass index; SBP, systolic blood pressure; DBP, diastolic blood pressure; Scr, serum creatinine; TC, total cholesterol; TG, triglycerides; HDL-C, high-density lipoprotein cholesterol; LDL-C, low-density lipoprotein cholesterol; FPG, fasting plasma glucose; ALT, alanine aminotransferase; UA, uric acid; RWT, relative wall thickness; LVM, left ventricular mass; LVMI, LVM index; $\beta$, standard $\beta$-coefficient; NT-proBNP, N-terminal pro-brain natriuretic peptide.

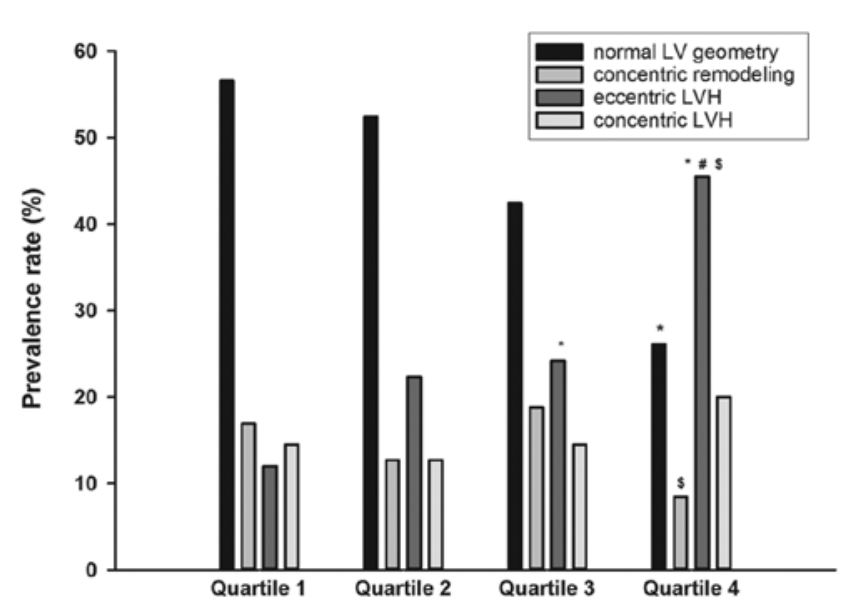

Figure 2. Association between $\mathrm{N}$-terminal pro-brain natriuretic peptide quartiles and LV geometric patterns in patients without heart failure. ${ }^{*} \mathrm{P}<0.05$ vs. quartile 1 ; ${ }^{\#} \mathrm{P}<0.05$ vs. quartile 2 ; ${ }^{\$} \mathrm{P}<0.05$ vs. quartile 3 . $\mathrm{LV}$, left ventricular; $\mathrm{LVH}$, left ventricular hypertrophy.

LVMI ( $\mathrm{P}<0.001$; Fig. 4), while an inverse correlation between Log(NT-proBNP) and RWT was indicated (P<0.05; Fig. 5). Using the multivariate regression model, significant associations between Log(NT-proBNP) and LVM, LVMI and RWT were confirmed after adjustment for age, sex, hypertension, PCI, diabetes, smoking history, HR, BMI, SBP, DBP, Scr, TC, TG, HDL-C, LDL-C, FPG, ALT and UA, as presented in Table III.

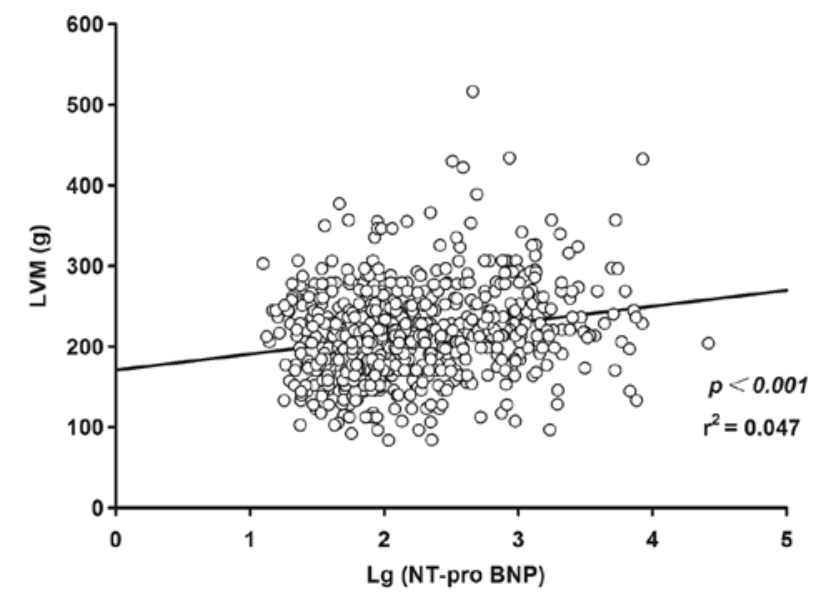

Figure 3. Correlation between $\log (\mathrm{NT}-$ proBNP) with LVM in patients without HF. Linear correlation analysis between $\log (\mathrm{NT}$-proBNP) and LVM [LVM=20.111xLog(NT-proBNP)+170.29]. NT-proBNP, N-terminal pro-brain natriuretic peptide; HF, heart failure; LVM, left ventricular mass.

\section{Discussion}

Accumulating studies suggest that inherent and acquired abnormalities within the natriuretic peptide system may contribute to the occurrence of a series of systemic and cardiac diseases, particularly cardiac hypertrophy (2). NT-proBNP is a BNP precursor released by ventricular tissues in response to an aggravated ventricular burden, which may induce ventricle 


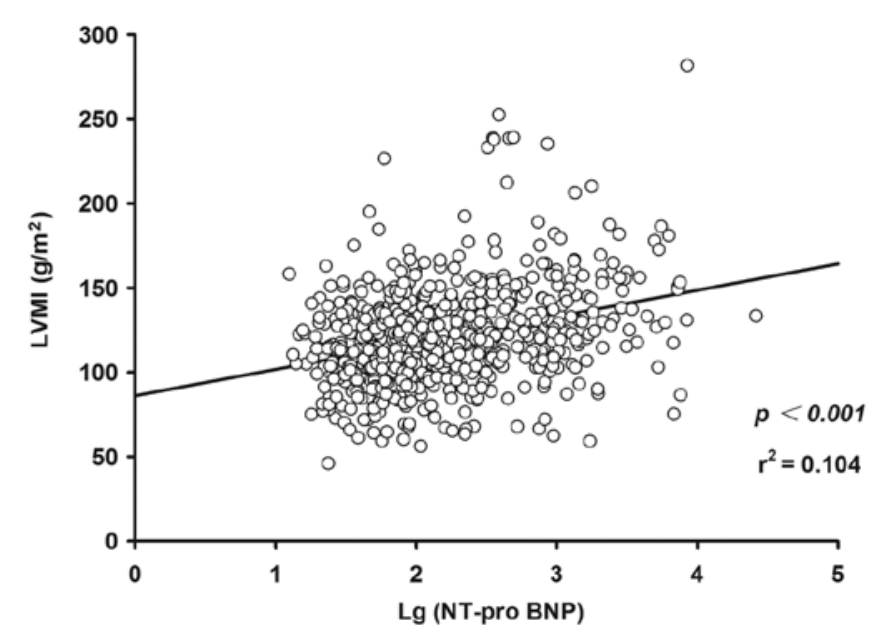

Figure 4. Correlation between Log(NT-proBNP) and LVMI in patients without HF. Linear correlation analysis between Log(NT-proBNP) and LVMI [LVMI=15.82x Log(NT-proBNP)+85.717]. NT-proBNP, N-terminal pro-brain natriuretic peptide; HF, heart failure: LVMI, left ventricular mass index.

remodeling. The preliminary NT-proBNP has a relatively large molecular size, as well as a longer half-life than the active form; therefore, its measurement is comparably convenient and is less likely to be interfered with by acute changes that may markedly affect the levels of other natriuretic peptides (15). As the relative stability of NT-proBNP enhances its reliability as a ventricular stress indicator and as a potential prognostic indicator, the serum levels of NT-proBNP were selected as the candidate biomarker to be investigated in the present study.

Previous studies have proved that NT-proBNP is an effective marker for LVH diagnosis. For instance, a population study indicated that NT-proBNP levels were elevated in LVH regardless of hypertension diagnosis (6). NT-proBNP measurements were also proved to be significant to $\mathrm{LVH}$ diagnosis for patients in the 1st year after renal transplantation (16) and were correlated with left ventricular dysfunction in dialysis patients (17). The present study provided an in-depth insight into the risk of LVH predicted by NT-proBNP quartiles and the complex association of NT-proBNP with LVM, LVMI and RWT in HF-negative patients. The results indicated that the prevalence rate of LVH increased progressively across individual NT-proBNP quartiles in patients without HF. The prevalence rate of abnormal LV geometric patterns was highest in the concentric remodeling group in NT-proBNP quartile 1 and in the eccentric LVH group in quartiles 2-4. According to univariate logistic regression, patients in NT-proBNP quartile 4 and quartile 3 had a 5.254- and a 1.757-fold increased LVH risk compared to patients in NT-proBNP quartile 1, respectively. Multivariate logistic regression also indicated that, compared with that of patients in NT-proBNP quartile 1, patients in NT-proBNP quartiles 2-4 had a significantly increased LVH risk. Furthermore, significant positive linear correlations of NT-proBNP with LVM and LVMI were identified, while an inverse correlation of NT-proBNP with RWT was indicated. After adjustments with the consideration of multiple potential interferences, including clinical parameters and predisposing conditions, significant associations between NT-proBNP and LVM, LVMI and RWT were further confirmed.

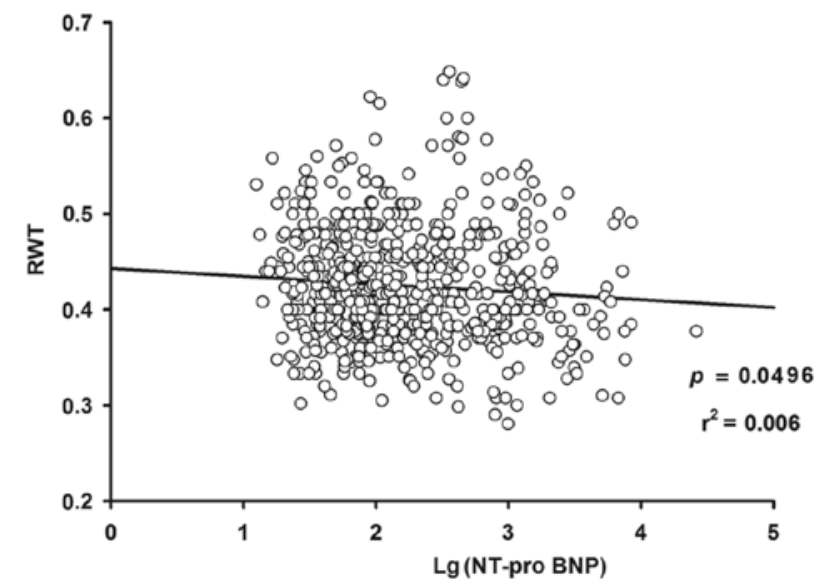

Figure 5. Correlation between $\log (\mathrm{NT}$-proBNP) and RWT in patients without HF. Linear correlation analysis between $\log (\mathrm{NT}$-proBNP) and RWT [RWT $=-0.008 x \operatorname{Lg}(\mathrm{NT}$-proBNP)+0.4419]. NT-proBNP, N-terminal pro-brain natriuretic peptide; HF, heart failure; RWT, relative wall thickness.

Numerous clinical studies have reported an association between upregulated natriuretic peptides and abnormally increased blood pressure. For instance, according to a cross-sectional study involving 202 participants with a previous history of dyspnea, the median NT-proBNP level was increased by $60 \%$ in hypertension-positive participants in comparison with hypertension-negative participants (18). A prospective analysis involving 1,801 participants reported that upregulated BNP plasma levels were significantly correlated with an elevated risk of aggravated hypertension occurring four years later for males rather than females (19). Bower et al (20) reported that participants in the lowest NT-proBNP quartile had a minimum risk of hypertension in comparison with any of the upper quartiles. In detail, the hypertension risks in the order from the lowest to the highest NT-proBNP quartiles were 1.00 at baseline (reference), 1.10 (95\% CI: 0.97-1.24), 1.08 (95\% CI: 0.95-1.24), and 1.24 (1.08-1.42), respectively. It also indicated that the log-unit increase of NT-proBNP corresponded to an $8 \%$ increase of the hypertension risk (95\% CI: 1.03-1.13) (20). In addition, hypertension was generally considered to be the predominant predisposing factor for LVH, which was suggested to be the result of excessive left ventricular afterload. Thus, it may be speculated that individuals with upregulated NT-proBNP were at increased risk of LVH, which may be associated with hypertension.

Accumulating studies suggested that inflammatory reactions may have a critical influence on the pathophysiological mechanisms of LVH $(21,22)$. Animal studies also indicated a critical role of inflammatory cytokines in the pathogenesis of LVH. Zhao et al (23) reported that genetic deletion of interleukin (IL)-6 attenuates transverse aortic constriction-induced LVH and LV dysfunction in mice. According to previous research, in a population of asymptomatic patients with high blood pressure, upregulated BNP was correlated with increased levels of inflammatory cytokines, including tumor necrosis factor- $\alpha$, IL-6 and IL-8 and also associated with increased LVMI and left atrial volume index (24). Furthermore, according to certain animal studies, BNPs have important roles in the regulation of myocardial fibrosis $(25,26)$. Excessive interstitial fibrosis was observed even in patients at the early stage of hypertension with only moderately upregulated LVH $(27,28)$. In addition, certain 
studies have reported that regression of the fibrosis degree resulted in improved LV function $(29,30)$. Thus, it may be speculated that the natriuretic peptide levels are clinically significant markers for the preliminary subclinical pathological process involving inflammation, myocardial fibrosis and cardiac remodeling.

In conclusion, the present study examined the association between NT-proBNP and LVH risk and the association between NT-proBNP and LVH hallmarks in patients without HF. Therefore, whether NT-proBNP levels have potential diagnostic, prognostic and epidemiological implications regarding $\mathrm{LVH}$ in patients without $\mathrm{HF}$ still requires in-depth investigation by further studies. However, the present study had several limitations. First, the study was observational. Furthermore, only patients who were admitted to the cardiology department were enrolled and due to lack of their NT-proBNP data, no healthy subjects were included for reference. More importantly, the sample size was limited and the results of the present study require further verification by studies with an extended scope.

\section{Acknowledgements}

Not applicable.

\section{Funding}

No funding was received.

\section{Availability of data and materials}

All data generated or analyzed during the present study are included in this published article.

\section{Authors' contributions}

LH and XW designed the study and performed the experiments. JZ and JY collected the data, LH and LFH analyzed the data and LH and XW prepared the manuscript. All authors read and approved the final manuscript.

\section{Ethics approval and consent to participate}

As the present study was a retrospective observational study, the requirement for patient consent and specifically ethical approval was waived.

\section{Patient consent for publication}

Not applicable.

\section{Competing interests}

The authors declare that they have no competing interests.

\section{References}

1. de Lemos JA, McGuire DK and Drazner MH: B-type natriuretic peptide in cardiovascular disease. Lancet 362: 316-322, 2003.

2. Gupta DK and Wang TJ: Natriuretic peptides and cardiometabolic health. Circ J 79: 1647-1655, 2015.
3. Santaguida PL, Don-Wauchope AC, Oremus M, McKelvie R, Ali U, Hill SA, Balion C, Booth RA, Brown JA, Bustamam A, et al: $\mathrm{BNP}$ and NT-probNP as prognostic markers in persons with acute decompensated heart failure: A systematic review. Heart Fail Rev 19: 453-470, 2014.

4. Ponikowski P, Voors AA, Anker SD, Bueno H, Cleland JG, Coats AJ, Falk V, González-Juanatey JR, Harjola VP, Jankowska EA, et al: 2016 ESC guidelines for the diagnosis and treatment of acute and chronic heart failure. Kardiol Pol 74: 1037-1147, 2016 (In Polish).

5. Morillas P, Castillo J, Quiles J, Nuñez D, Guillén S, Maceira A, Rivera $\mathrm{M}$ and Bertomeu V: Usefulness of NT-probNP level for diagnosing left ventricular hypertrophy in hypertensive patients. A cardiac magnetic resonance study. Rev Esp Cardiol 61: 972-975, 2008 (In English, Spanish).

6. Rivera Otero JM, Taléns-Visconti R, Salvador A, Bertomeu V, Miró V, Jordán A, Sogorb F, Cortés R, Payá R, Diago JL, et al: Ventricular hypertrophy increases NT-proBNP in subjects with and without hypertension. Int J Cardiol 96: 265-271, 2004.

7. Parekh $\mathrm{N}$ and Maisel AS: Utility of $\beta$-natriuretic peptide in the evaluation of left ventricular diastolic function and diastolic heart failure. Curr Opin Cardiol 24: 155-160, 2009.

8. Lubien E, DeMaria A, Krishnaswamy P, Clopton P, Koon J, Kazanegra R, Gardetto N, Wanner E and Maisel AS: Utility of B-natriuretic peptide in detecting diastolic dysfunction: Comparison with doppler velocity recordings. Circulation 105: 595-601, 2002.

9. Santosa YP, Tjandrawati A, Noormartany, Erwinanto, Yahya AF, Martanto E, Tedjokusumo P, Purnomowati A and Antono E: Comparison of pro $\beta$-natriuretic peptide in hypertensive patients with and without diastolic dysfunction. Acta Med Indones 40: 19-23, 2008.

10. Chinese Society of Cardiology of Chinese Medical Association; Editorial Board of Chinese Journal of Cardiology: Chinese guidelines for the diagnosis and treatment of heart failure 2014 . Zhonghua Xin Xue Guan Bing Za Zhi 42: 98-122, 2014 (In Chinese).

11. Lang RM, Bierig M, Devereux RB, Flachskampf FA, Foster E, Pellikka PA,Picard MH, Roman MJ, Seward J, Shanewise JS, et al: Recommendations for chamber quantification: A report from the American society of echocardiography's guidelines and standards committee and the chamber quantification writing group, developed in conjunction with the European association of echocardiography, a branch of the European society of cardiology. J Am Soc Echocardiogr 18: 1440-1463, 2005.

12. Liu LS; Writing Group of Chinese Guidelines for the Management of Hypertension: 2010 Chinese guidelines for the management of hypertension. Zhonghua Xin Xue Guan Bing Za Zhi 39: 579-615, 2011 (In Chinese).

13. Gebker R, Mirelis JG, Jahnke C, Hucko T, Manka R, Hamdan A, Schnackenburg B, Fleck E and Paetsch I: Influence of left ventricular hypertrophy and geometry on diagnostic accuracy of wall motion and perfusion magnetic resonance during dobutamine stress. Circ Cardiovasc Imaging 3: 507-514, 2010.

14. Huang L, Teng T, Bian B, Yao W, Yu X, Wang Z, Xu Z and Sun Y: Zinc levels in left ventricular hypertrophy. Biol Trace Elem Res 176: 48-55, 2017.

15. Yamazaki M, Ogawa T, Tamei N, Ando Y and Nitta K: Relation of N-terminal pro-B-type natriuretic peptide (NT-proBNP) and left atrial volume index to left ventricular function in chronic hemodialysis patients. Heart Vessels 26: 421-427, 2011.

16. Slubowska K, Sadowska A, Kwiatkowski A and Durlik M: N-terminal pro-B-type natriuretic peptide (NT-proBNP) assessment in the first year after renal transplantation and its relationship with graft function and left ventricular hypertrophy. Transplant Proc 46: 2729-2732, 2014.

17. David S, Kümpers P, Seidler V, Biertz F, Haller H and Fliser D: Diagnostic value of $\mathrm{N}$-terminal pro-B-type natriuretic peptide (NT-proBNP) for left ventricular dysfunction in patients with chronic kidney disease stage 5 on haemodialysis. Nephrol Dial Transplant 23: 1370-1377, 2008.

18. Rivera M, Taléns-Visconti R, Salvador A, Bertomeu V, Miró V, García de Burgos F, Climent V, Cortés R, Payá R, Pérez-Boscá JL, et al: NT-proBNP levels and hypertension. Their importance in the diagnosis of heart failure. Rev Esp Cardiol 57: 396-402, 2004 (In Spanish).

19. Freitag MH, Larson MG, Levy D, Benjamin EJ, Wang TJ, Leip EP, Wilson PW and Vasan RS; Framingham Heart Study: Plasma brain natriuretic peptide levels and blood pressure tracking in the framingham heart study. Hypertension 41: 978-983, 2003. 
20. Bower JK, Lazo M, Matsushita K, Rubin J, Hoogeveen RC, Ballantyne CM and Selvin E: N-terminal pro-brain natriuretic peptide (NT-proBNP) and risk of hypertension in the atherosclerosis risk in communities (ARIC) study. Am J Hypertens 28 : 1262-1266, 2015.

21. Masiha S, Sundstrom J and Lind L: Inflammatory markers are associated with left ventricular hypertrophy and diastolic dysfunction in a population-based sample of elderly men and women. J Hum Hypertens 27: 13-17, 2013.

22. Erten Y, Tulmac M, Derici U, Pasaoglu H, Altok Reis K, Bali M, Arinsoy T, Cengel A and Sindel S: An association between inflammatory state and left ventricular hypertrophy in hemodialysis patients. Ren Fail 27: 581-589, 2005.

23. Zhao L, Cheng G, Jin R, Afzal MR, Samanta A, Xuan YT, Girgis M, Elias HK, Zhu Y, Davani A, et al: Deletion of interleukin-6 attenuates pressure overload-induced left ventricular hypertrophy and dysfunction. Circ Res 118: 1918-1929, 2016.

24. Phelan D, Watson C, Martos R, Collier P, Patle A, Donnelly S, Ledwidge M, Baugh $\mathrm{J}$ and McDonald $\mathrm{K}$ : Modest elevation in BNP in asymptomatic hypertensive patients reflects sub-clinical cardiac remodeling, inflammation and extracellular matrix changes. PLoS One 7: e49259, 2012.

25. Kapoun AM, Liang F, O'Young G, Damm DL, Quon D, White RT, Munson K, Lam A, Schreiner GF and Protter AA: B-type natriuretic peptide exerts broad functional opposition to transforming growth factor-beta in primary human cardiac fibroblasts: Fibrosis, myofibroblast conversion, proliferation, and inflammation. Circ Res 94: 453-461, 2004.
26. Tamura N, Ogawa Y, Chusho H, Nakamura K, Nakao K, Suda M, Kasahara M, Hashimoto R, Katsuura G, Mukoyama M, et al: Cardiac fibrosis in mice lacking brain natriuretic peptide. Proc Natl Acad Sci USA 97: 4239-4244, 2000.

27. Rossi MA: Pathologic fibrosis and connective tissue matrix in left ventricular hypertrophy due to chronic arterial hypertension in humans. J Hypertens 16: 1031-1041, 1998.

28. Ciulla M, Paliotti R, Hess DB, Tjahja E, Campbell SE, Magrini F and Weber KT: Echocardiographic patterns of myocardial fibrosis in hypertensive patients: Endomyocardial biopsy versus ultrasonic tissue characterization. J Am Soc Echocardiogr 10: 657-664, 1997.

29. Diez J, Querejeta R, López B, González A, Larman M and Martínez Ubago JL: Losartan-dependent regression of myocardial fibrosis is associated with reduction of left ventricular chamber stiffness in hypertensive patients. Circulation 105: 2512-2517, 2002

30. Brilla CG, Funck RC and Rupp H: Lisinopril-mediated regression of myocardial fibrosis in patients with hypertensive heart disease. Circulation 102: 1388-1393, 2000.

(i) (9) This work is licensed under a Creative Commons Attribution-NonCommercial-NoDerivatives 4.0 International (CC BY-NC-ND 4.0) License. 